\title{
AXISYMMETRIC STOKES FLOW IMAGES IN SPHERICAL FREE SURFACES WITH APPLICATIONS TO RISING BUBBLES
}

\author{
J. F. HARPER'
}

(Received 25 August 1982)

\begin{abstract}
A theorem is derived for the hydrodynamic image of an axially symmetric slow viscous (Stokes) flow in a sphere which is impermeable and free of shear stress. A second theorem establishes a sense in which such a flow past an arbitrary rigid surface or shear-free sphere becomes, on inversion in an arbitrary sphere with its centre on the axis of symmetry, a flow past the rigid or shear-free inverse of that surface or sphere.

The theorems are used to simplify the proofs of a number of known results for images of point singularities in plane and spherical rigid and free boundaries, and for a pair of bubbles rising steadily in line in a viscous fluid. They also give for the first time accurate numerical solutions for the velocities of each of a larger number of spherical bubbles rising quasi-steadily in line. These enable one to assess the accuracy of simple approximations to those velocities.
\end{abstract}

\section{Introduction}

Spheres on which there is no shear stress are found as boundaries to slow viscous (Stokes) flow in two important contexts. The Earth's core is, to a good approximation, such a boundary for the convection in its mantle, and the surface of a small gas bubble rising in an uncontaminated viscous fluid is such a boundary for the flow outside it. In the two cases the spherical shape is maintained by gravity and by surface-tension forces [13, 14, 16], but tangential motion is unimpeded.

Theorem 1 and a lemma give the image systems in such surfaces. They are simpler than the corresponding known results $[4,5,9,10,20,23]$ for rigid spheres. Theorem 2 shows how one can invert an axially symmetric configuration of

\footnotetext{
'Department of Mathematics, Victoria University of Wellington, Private Bag, Wellington, New Zealand.

(C) Copyright Australian Mathematical Society 1983, Serial-fee code 0334-2700/83.
} 
shear-free spheres or rigid surfaces of general shape, in any sphere and with its centre on the axis, and relate a Stokes flow bounded by one configuration to one bounded by the other.

The theorems permit simpler proofs to be given for a number of known results: images of axisymmetric point singularities (Stokeslets, Stokes-multipoles and potential-multipoles) in free or rigid spherical or plane surfaces, the flow past one bubble rising towards a plane free surface, or two bubbles rising steadily in line. Departures from the exact plane or spherical shape will occur in practice, of course $[1,3,17,18]$; they will be small for sufficiently slow motions and are not considered in this paper. A new problem which can be solved is the quasi-steady rise of several bubbles in a vertical line. Two different approximations have been given previously: ignoring all but nearest-neighbour interactions [19], and ignoring all but first-order interactions [22]. Numerical solutions for the velocity of each bubble show the superiority of the latter approximation. Geophysical applications of the results also exist, but will be discussed elsewhere.

\section{The sphere theorems}

THEOREM 1. If $\psi(r, \theta)$ is the Stokes stream function of an axially symmetric steady slow viscous (Stokes) flow with no singularities on the sphere $r=a$, then so is

$$
\psi_{1}(r, \theta)=\psi(r, \theta)-\left(r^{3} / a^{3}\right) \psi\left(a^{2} / r, \theta\right)
$$

$(r, \theta)$ being spherical polar coordinates; and the fluid motion described by $\psi_{1}$ has the sphere $r=a$ as a shear-free stream-surface, for which

$$
\psi_{1}=0 \text { and }(\partial / \partial r)\left(r^{-2} \partial \psi_{1} / \partial r\right)=0
$$

Proof. It is already known [9] that $\psi_{1}$ obeys the relevant equation of motion, i.e.,

$$
L \psi_{1} \equiv\left\{\frac{\partial^{2}}{\partial r^{2}}+\frac{1-\mu^{2}}{r^{2}} \frac{\partial^{2}}{\partial \mu^{2}}\right\}^{2} \psi_{1}=0
$$

where $\mu=\cos \theta$. To show that $\psi_{1}$ also satisfies the boundary conditions is a straightforward exercise in differentiation. The first equation (2) makes the sphere a stream-surface; the second makes the tangential shear stress zero on it $[13$, page $125-8]$. 
DEFINITION. For any flow with stream-function $\psi(r, \theta)$ obeying equation (3), the inverse flow in the sphere $r=a$ is defined to be the flow with stream-function $\psi^{\prime}(r, \theta)$ given by

$$
\psi^{\prime}(r, \theta)=-\left(r^{3} / a^{3}\right) \psi\left(a^{2} / r, \theta\right)=-\left(r / r^{\prime}\right)^{3 / 2} \psi\left(r^{\prime}, \theta\right)
$$

where $r r^{\prime}=a^{2}$, and the singularities of $\psi^{\prime}(r, \theta)$ are defined to be the images in the sphere $r=a$ of the singularities of $\psi(r, \theta)$.

For this purpose we extend the term "singularity" to include axisymmetric flows non-vanishing at infinity and regular everywhere else, i.e. uniform streams, Stokesons, stressons and their derivatives [8].

REMARKS. The inverse of the inverse of a flow is the original flow, and the image of an image is the original singularity. Theorem 1 is the analogue for Stokes flow of Butler's sphere theorem [6] for inviscid flow.

LEMMA. For any flow with stream-function $\psi$ obeying equation (3) in a region including the sphere $r=a$, that sphere is a shear-free stream-surface $\psi=0$ if and only if the flow is its own inverse in the sphere.

Proof. The "only if" part is a restatement of Theorem 1; the "if" part is another exercise in differentiation.

The purpose of this lemma is to prove the less obvious part of the following inversion theorem.

THEOREM 2. Let $\psi_{1}(r, \theta)$ be the stream-function, obeying equation (3), of a flow in which either a sphere $S_{1}$ is a shear-free stream-surface on which $\psi_{1}=0$ and $\partial / \partial r_{1}\left(r_{1}^{-2} \partial \psi_{1} / \partial r_{1}\right)=0$, or any axisymmetric surface $R_{1}$ is a rigid boundary on which $\psi_{1}=0$ and $\partial \psi_{1} / \partial r_{1}=0$, where $r_{1}$ is the distance from the centre of $S_{1}$. Let the geometrical inverse of $S_{1}$ or $R_{1}$ in any sphere $T$ whose centre is on the axis of symmetry of $\psi_{1}$ be $S_{2}$ or $R_{2}$. Then the inverse flow of $\psi_{1}$ in $T$ has $S_{2}$ or $R_{2}$ as a shear-free or rigid boundary, respectively.

Proof. The rigid case is almost obvious, as the boundary condition $R_{1}$ is $\psi_{1}=0, \nabla \psi_{1}=0$, and the inverse flow must satisfy the same conditions on $R_{2}$. For the shear-free case it is sufficient, by the lemma, to show that if $\psi_{1}$ is its own inverse in $S_{1}$, then the inverse of $\psi_{1}$ in $T$ is its own inverse in $S_{2}$. That can be established geometrically. By axial symmetry, $S_{1}, S_{2}$ and $T$ have collinear centres. Let $A_{1}, B_{1}$ be any two points inverse with respect to $S_{1}$ whose centre is $O_{1}$, and let 


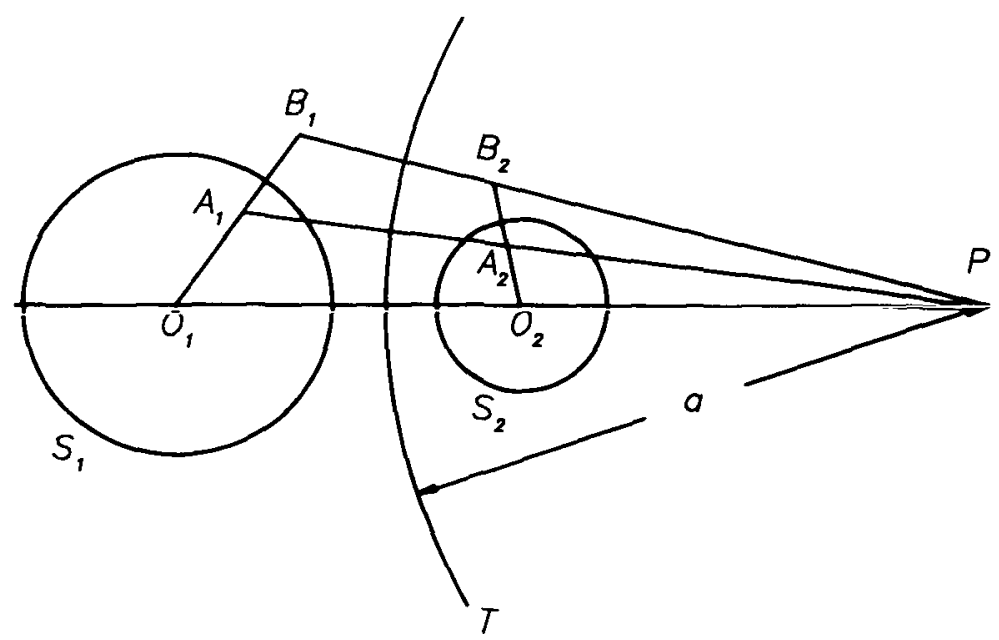

Figure 1. Configuration for the proof of Theorem 2.

$\psi_{1}$ be equal to $\psi_{1}\left(A_{1}\right), \psi_{1}\left(B_{1}\right)$ at those points. The self-inverse property of $\psi_{1}$ can then be written

$$
\psi_{1}\left(A_{1}\right)=-\left(O_{1} A_{1} / O_{1} B_{1}\right)^{3 / 2} \psi_{1}\left(B_{1}\right)
$$

If $A_{2}, B_{2}$ are the inverses of $A_{1}, B_{1}$ in $T$, and if $O_{2}, P$ are the centres of $S, T$, the inverse flow $\psi_{2}$ must obey

$$
\begin{aligned}
& \psi_{2}\left(A_{2}\right)=-\left(P A_{2} / P A_{1}\right)^{3 / 2} \psi_{1}\left(A_{1}\right), \\
& \psi_{2}\left(B_{2}\right)=-\left(P B_{2} / P B_{1}\right)^{3 / 2} \psi_{1}\left(B_{1}\right),
\end{aligned}
$$

(see Figure 1). From equations (5), (6), (7) we find that

$$
\frac{\psi_{2}\left(A_{2}\right)}{\psi_{2}\left(B_{2}\right)}=-\left\{\frac{P A_{2} \cdot O_{1} A_{1} \cdot P B_{1}}{P B_{1} \cdot O_{1} B_{1} \cdot P B_{2}}\right\}^{3 / 2} .
$$

Now $A_{2}$ is the inverse of $B_{2}$ in $S_{2}$ [21, Section 25.2] and the triangles $P A_{2} B_{2}$, $P B_{1} A_{1}$ are similar because $P A_{1} . P A_{2}=P B_{1} . P B_{2}$, and so

$$
\begin{aligned}
\frac{P A_{2} \cdot O_{1} A_{1} \cdot P B_{1}}{P A_{1} \cdot O_{1} B_{1} \cdot P B_{2}} & =\left(\frac{P B_{1}}{P A_{1}}\right)^{2} \frac{O_{1} A_{1}}{O_{1} B_{1}}=\left(\frac{P A_{2}}{P B_{2}}\right)^{2} \frac{O_{1} A_{1}}{O_{1} B_{1}} \\
& =\frac{\sin \left(O_{1} P A_{1}\right) \sin \left(A_{1} B_{1} P\right)}{\sin \left(O_{1} P B_{1}\right) \sin \left(B_{1} A_{1} P\right)}=\frac{O_{2} A_{2}}{O_{2} B_{2}},
\end{aligned}
$$

because of the equal angles of the similar triangles already mentioned. Hence

$$
\frac{\psi_{2}\left(A_{2}\right)}{\psi_{2}\left(B_{2}\right)}=-\left\{\frac{O_{2} A_{2}}{O_{2} B_{2}}\right\}^{3 / 2},
$$


which is the required self-inverse property for $\psi_{2}$ in the sphere $S_{2}$.

REMARK. The sphere $T$ need have no simple relation to the flows: it merely transforms boundaries.

\section{Images of singularities}

The inverse flow of the uniform stream $\psi=\frac{1}{2} U r^{2} \sin ^{2} \theta$ in the sphere $r=a$ has the stream-function $-\frac{1}{2} U a r \sin ^{2} \theta$. This is the flow due to a Stokeslet at the centre, of strength $F=4 \pi U a \eta$, where $\eta$ is the viscosity of the fluid and $F$ is a force pointing upstream. The classical Rybczynski-Hadamard flow [13] past a shear-free sphere is of course the superposition of the uniform stream and its inverse, and $F$ is the force which is exerted by the sphere on the fluid.

Now consider a Stokeslet at $r=s_{1}, \theta=0$. With $\cos \theta=\mu$ as before, its stream-function is

$$
\psi(r, \theta)=-\frac{F r^{2}\left(1-\mu^{2}\right)}{8 \pi \eta\left(r^{2}-2 \mu r s_{1}+s_{1}^{2}\right)^{1 / 2}},
$$

and if $s_{2}=a^{2} / s_{1}$,

$$
-\left(r^{3} / a^{3}\right) \psi\left(a^{2} / r, \theta\right)=\frac{F^{2}\left(1-\mu^{2}\right)}{8 \pi \eta s_{1}\left(r^{2}-2 \mu r s_{2}+s_{2}^{2}\right)^{1 / 2}} .
$$

The image of Stokeslet $F$ at $s_{1}$ is thus a Stokeslet $-F a / s_{1}=-F s_{2} / a$ at the inverse point $s_{2}$. It does not matter which one is inside the sphere; one points at the centre, the other away, and the one inside has the smaller strength. It is the simplicity of the result and the absence of higher-order singularities that enables one to use images for problems involving rising bubbles much more easily than for rigid spheres.

However, Theorem 2 also applies to rigid boundaries, and it is of interest to see what the images of higher-order point singularities are. These singularities are axisymmetric Stokes multipoles with stream functions of the form

$$
{ }_{s} \psi_{n}(r, \mu)=r^{-(n-3)}\left\{P_{n}(\mu)-P_{n-2}(\mu)\right\} \quad \text { for } n=2,3,4, \ldots,
$$

of which the Stokeslet is the case $n=2$, and axisymmetric potential multipoles of the form

$$
{ }_{p} \psi_{n}(r, \mu)=r^{-(n-1)}\left\{P_{n}(\mu)-P_{n-2}(\mu)\right\} \text { for } n=2,3,4, \ldots,
$$

of which the potential doublet is the case $n=2$. Here $P_{n}$ denotes the Legendre polynomial, and our normalisation differs from the usual one [8] in the interests of algebraic simplicity. 
If any of these singularities is at the centre of a sphere, its image is an appropriate and easily found singularity "at infinity". We give here the images of singularities elsewhere. We begin with the image in $r=a$ of the function $\psi=r_{1}^{-\beta} P_{\alpha}\left(\mu_{1}\right)$ (see Figure 2), where $\mu_{i}=\cos \theta_{t}$ for $i=1,2$, and $s_{1} s_{2}=a^{2}$. Because $r_{i}^{2}=r^{2}-2 r s_{i} \mu+s_{i}^{2}$ and $r \mu=s_{i}+r_{i} \mu_{t}$, we find that with $\psi$ as above, its inverse $\psi^{\prime}$ in $r=a$ is

$$
\psi^{\prime}=\frac{r^{3+\beta-\tilde{\alpha}}}{a^{3} s_{1}^{\beta}}(-1)^{\alpha} r_{2}^{-\beta} r^{\alpha} P_{\alpha}\left[\cos \left(\theta_{2}-\theta\right)\right]
$$

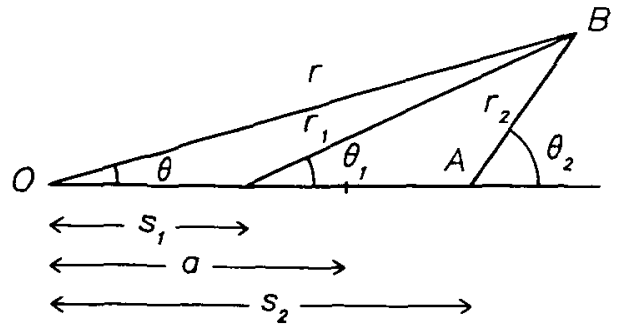

Figure 2. Definition sketch for the coordinate systems used in Section 3.

Imagining for the moment that $A B$ is a fixed symmetry axis in Figure 2, and $O$ is a variable point, we see that $r^{\alpha} P_{\alpha}\left[\cos \left(\theta_{2}-\theta\right)\right]$ is a spherical harmonic of degree $n$, which can be written as

$$
r^{\alpha} P_{\alpha}\left[\cos \left(\theta_{2}-\theta\right)\right]=\sum_{k=0}^{\alpha} \frac{\alpha ! s_{2}^{k} r_{2}^{\alpha-k} P_{k}\left(\mu_{2}\right)}{k !(\alpha-k) !}
$$

because if $\mu_{2}= \pm 1$ it is $r^{\alpha}=\left(r_{2} \pm s_{2}\right)^{\alpha}$ respectively. Equations (12), (13) and $r^{2}=r_{2}^{2}+2 r_{2} s_{2} \mu_{2}+s_{2}^{2}$, with the recurrence relations for Legendre polynomials, then enable us to write the inverses ${ }_{s} \psi_{n}^{\prime}$ and ${ }_{p} \psi_{n}^{\prime}$ in the sphere $r=a$ of the stream-functions ${ }_{s} \psi_{n}$ and ${ }_{p} \psi_{n}$ as

$$
\begin{gathered}
{ }_{s} \psi_{n}^{\prime}=\frac{(-1)^{n+1} s_{2}^{n-3}}{a^{2 n-3}} \sum_{N=2}^{n} \frac{(n-2) !(2 n-1) s_{2}^{N}}{(N-2) !(n-N) !(2 N-1)}{ }_{s} \psi_{N}\left(r_{2}, \mu_{2}\right), \\
{ }_{p} \psi_{n}^{\prime}=\frac{r^{2} s_{1}^{2}}{r_{2}^{2}}{ }_{s} \psi_{n}^{\prime} .
\end{gathered}
$$

Equation (14) gives the image of a Stokes-multipole of order $n$ in terms of a sum of Stokes-multipoles'of all orders from 2 to $n$ at the inverse point. Because

$$
\frac{r^{2}}{r_{2}^{2}} \psi_{N}={ }_{s} \psi_{N}+2 s_{2}\left(\frac{N+1}{2 N+1} s_{N+1}+\frac{N-2}{2 N-3} \psi_{N-1}\right)+s_{2 p}^{2} \psi_{N}
$$


equations (15) and (16) can be used also to express the image of a potential multipole of order $n$ in terms of both potential and Stokes multipoles at the inverse point of orders up to $n$ and $n+1$. For example, the flow of a uniform stream past a rigid sphere is well known to require a Stokeslet and a potentialdoublet at the centre. Inverting with respect to any point on the axis of symmetry gives Nigam and Srinivasan's result [20] for a Stokeslet pointing at a rigid sphere; if the point of inversion is also on the sphere we get Blake and Chwang's result [4] for a Stokeslet pointing at a rigid plane wall. The present method would evidently produce the corresponding results for higher orders but with rather large amounts of algebra. They are already known [20] for the potential source and doublet.

Images of point sources can also be found, but they are not point singularities. If, for example, $\psi=0$ on the side $\theta=0$ of the source, a source solution ${ }_{p} \psi_{1}\left(r_{1}, \mu_{1}\right)=\mu_{1}-1$ has $\eta=-2$ on the other side, and $r^{3} / a_{p}^{3} \psi_{1}=-2 r^{3} / a^{3}$ on the inverse of that far side. That is why a line distribution of sources appears in Nigam and Srinivasan's solution.

\section{A pair of bubbles rising in a viscous fluid}

Some years ago Wacholder and Weihs [24] solved three problems concerning spherical drops or bubbles moving under gravity:

a) two of them rising in line, possibly with different radii and internal viscosities;

b) one drop or bubble moving vertically towards or away from a horizontal rigid surface;

c) one drop or bubble moving vertically towards or away from a horizontal free surface.

The solutions involved elaborate calculations with infinite series of Legendre polynomials in bispherical coordinates, as did a more recent investigation of a rigid sphere rotating or translating in any direction near a free surface [18].

In the special case of gas bubbles of negligibly small internal viscosity, in a liquid with surface tension high enough to keep their surfaces spherical [16], but with negligibly small surface tension gradients [14], we give simpler routes to Wacholder and Weihs' results for cases a) and c). Unfortunately the image systems are far less simple when the surfaces are other than tangentially stress-free.

A single bubble of radius $a$ moving at speed $U$ in unbounded viscous fluid has $\psi=\frac{1}{2} U R^{2} / r$ in a coordinate system at rest relative to the distant fluid, where $R, r$ are distances from the axis and the centre; $\psi$ is, of course, the flow due to a Stokeslet of strength $F=-4 \pi \eta U a$ at the centre of the bubble. For two bubbles of the same or different radii rising in the same vertical line, the flow is that due to:

(1) the two central Stokeslets;

(2) the image of each in the other bubble; 
(3) the images of those images in the original bubbles, and so on.

We give further detail only for the case of two equal bubbles, though it could be calculated for unequal ones also. If the $n$th order images are $F_{n}$ at distance $d_{n}$ below the centre of the upper bubble and above the centre of the lower one, and if the radius of each bubble is $a$, and their centres are a distance $b$ apart, then equation (9) gives

$$
\begin{gathered}
F_{0}=F \text { and } d_{0}=0, \\
F_{n+1}=-F_{n} \frac{d_{n+1}}{a}=-F_{n} \frac{a}{b-d_{n}} \text { for } n \geqslant 0,
\end{gathered}
$$

and the stream function $\psi$ for the flow as a whole is

$$
\psi=R^{2} \sum_{n=0}^{\infty}\left(\frac{1}{r_{n 1}}+\frac{1}{r_{n 2}}\right),
$$

where $r_{n 1}, r_{n 2}$ are the distances from a point in the flow field to the $n$th image Stokeslet in the upper and lower bubbles (see Figure 3). The total drag force on each bubble is the total strength of all the Stokeslets inside it, i.e.,

$$
G=F \Lambda(\tau)=F\left(1-\varepsilon_{1}+\varepsilon_{1} \varepsilon_{2}-\varepsilon_{1} \varepsilon_{2} \varepsilon_{3}+\cdots\right)
$$

where $\varepsilon_{n}=d_{n} / a, \tau=a / b$, the ratio of bubble radius to distance between centres, and

$$
\begin{aligned}
& \varepsilon_{0}=0, \\
& \varepsilon_{n}=\frac{\tau}{1-\tau \varepsilon_{n-1}} \quad \text { for } n \geqslant 1 .
\end{aligned}
$$

As $\tau$ increases from 0 (bubbles infinitely far apart) to $\frac{1}{2}$ (touching), $\Lambda(\tau)$ decreases from 1 to $\ln 2=0.693 \cdots$ and is the proportional drag reduction on either bubble due to the presence of the other. It is the same function as $\lambda(1 / 2 \tau, 0)$ of Wacholder and Weihs [24], but expressed in a simpler series: a programmable pocket calculator sufficed to obtain Table 1. Figure 3 shows the flow pattern for the extreme (and unrealistic) case of touching bubbles; it will be close to the actual pattern for bubbles very near to each other if they remain practically spherical, i.e., if viscous stresses are much smaller than those due to surface tension, i.e., $\eta U \ll \sigma$, where $\sigma$ is the surface tension [17].

Table 1 also gives values of $1 / \Lambda(\tau)$, which is the speed of each bubble divided by the speed in the absence of the other if, as usual, gravity provides the force on each irrespective of the position of the other. It will be seen that it is very close 
indeed to $1+\tau$, being only $4 \%$ below that value for touching bubbles. The Taylor series for $1 / \lambda(\tau)$ can be found from equations (8) and (9) as

$$
1 / \Lambda(\tau)=1+\tau-\tau^{4}-\tau^{6}+2 \tau^{7}+\cdots .
$$

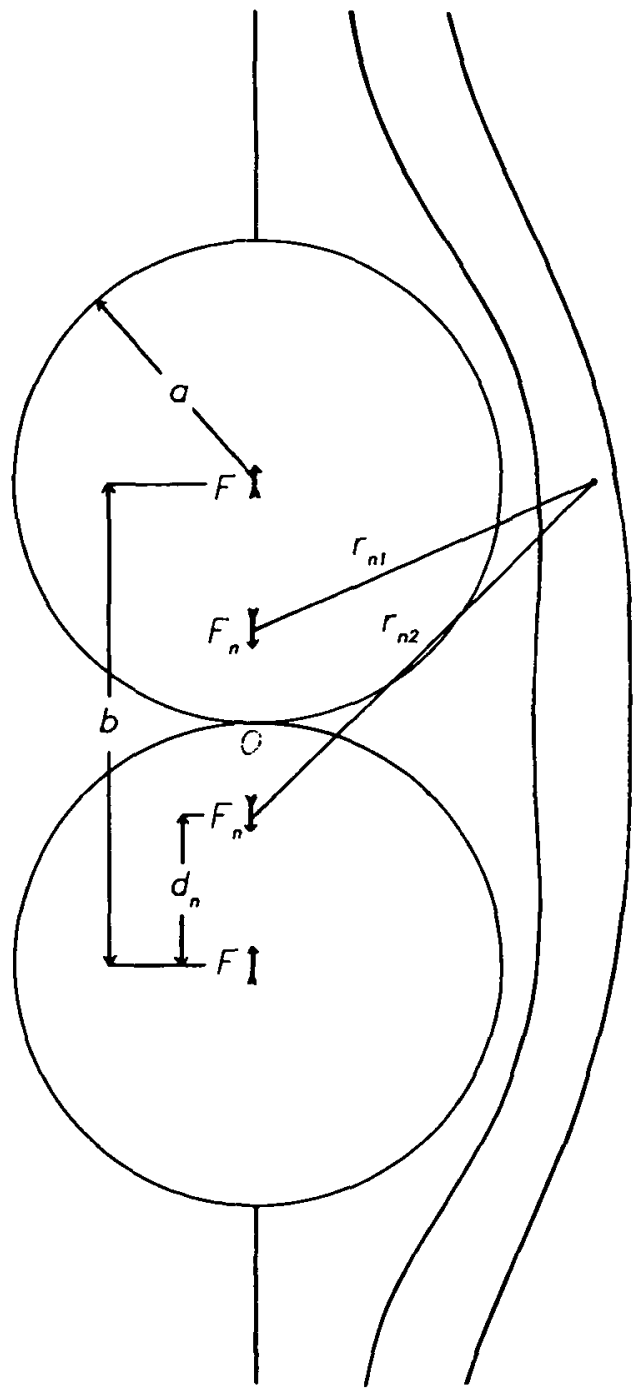

Figure 3. A pair of rising bubbles, with the streamline pattern for the case $b=2 a$, calculated by using Theorem 2 to invert with respect to $O$, so that the bubbles transform to parallel planes, the stream at infinity to a Stokeslet at $O$, with image Stokeslets equally spaced, alternately up and down, and all the same strengths, and then using the Fourier series [12, page 445], which was Cesàro-summed to improve convergence. 
TABLE 1. Values of $\tau, \alpha$ and $\Lambda(\tau)$ both for a pair of bubbles $(\tau>0)$ and for a bubble and a plane free surface $(\tau<0)$.

\begin{tabular}{clll}
$\tau$ & $\alpha=\cosh ^{-1}(1 / 2|\tau|)$ & \multicolumn{1}{c}{$\Lambda(\tau)$} & $1 / \Lambda(\tau)$ \\
\hline 0.5 & 0.0 & $0.693147=\ln 2$ & 1.442695 \\
0.4 & $0.693147=\ln 2$ & 0.727649 & 1.374289 \\
0.3 & $1.098612=\ln 3$ & 0.774205 & 1.291647 \\
0.2 & 1.566799 & 0.834473 & 1.198361 \\
0.1 & 2.292432 & 0.793174 & $i .0998 \hat{9} \hat{9}$ \\
0 & $\infty$ & 1.0 & 1.0 \\
-0.1 & 2.292432 & 1.111236 & 0.899899 \\
-0.2 & 1.566799 & 1.252656 & 0.798304 \\
-0.3 & $1.098612=\ln 3$ & 1.448289 & 0.690470 \\
-0.4 & $0.693147=\ln 2$ & 1.778396 & 0.562304 \\
-0.45 & 0.467145 & 2.109325 & 0.474085 \\
-0.5 & 0.0 & $\infty$ & 0
\end{tabular}

In this expression the coefficients are all integers, the radius of convergence is $\frac{1}{2}$, and the high quality of $1+\tau$ as an approximation is due to the vanishing of the $\tau^{2}$ and $\tau^{3}$ terms. In physical terms, the $1+\tau$ approximation says that to find the velocity of either bubble in the presence of the other, add to its velocity $V$ when alone in the fluid the velocity $V_{\tau}$ induced by the Rybczynski-Hadamard Stokeslet at the centre of the other.

For general $\tau$, there is a closed-form expansion for $\Lambda(\tau)$ obtained as follows. Equation (21) implies that

$$
\varepsilon_{n}=\tau p_{n-1}(x) / p_{n}(x),
$$

where $p_{n}$ is a polynomial in the variable $x=\tau^{2}$, of degree $\frac{1}{2} n$ for even $n$ and $\frac{1}{2}(n-1)$ for odd $n$, with the linear recurrence relation

$$
\begin{aligned}
& p_{0}(x)=p_{1}(x)=1, \\
& p_{n+1}(x)-p_{n}(x)+x p_{n-1}(x)=0 \quad \text { for } n \geqslant 1 .
\end{aligned}
$$

This leads to

$$
p_{n}(x)=\left(A^{n+1}-B^{n+1}\right) /(1-4 x)^{1 / 2},
$$

where

$$
\begin{aligned}
& A=\frac{1}{2}\left\{1+\left(1-4 \tau^{2}\right)^{1 / 2}\right\}, \\
& B=\frac{1}{2}\left\{1-\left(1-4 \tau^{2}\right)^{1 / 2}\right\} .
\end{aligned}
$$

If $y=(B / A)^{1 / 2}=\tau / A=B / \tau=e^{-\alpha}$, equation (20) can be written

$$
\begin{aligned}
\Lambda(\tau) & =\frac{1-y^{2}}{y}\left(\frac{y}{1-y^{2}}-\frac{y^{2}}{1-y^{4}}+\frac{y^{3}}{1-y^{6}}-\frac{y^{4}}{1-y^{8}}+\cdots\right) \\
& ={ }_{2} \Phi_{1}\left(y^{2}, y^{2} ; y^{4} ;-y\right),
\end{aligned}
$$


[12, page 213], where ${ }_{2} \Phi_{1}$ is Heine's basic hypergeometric function, not to be confused with Gauss's hypergeometric function ${ }_{2} F_{1}$, [2]. The series (27) is, term by term, the same as the series in equation (20) and is no better for numerical work.

\section{A single bubble rising beneath a plane free surface}

If one spherical bubble is rising beneath a plane surface, both the sphere and the plane being tangentially stress-free, the calculations of the previous section are changed very little if $b$ is interpreted as the distance from the bubble centre to its reflection in the free surface. With $\tau, \varepsilon_{n}, A$ and $y$ defined as before, we find that the - signs in equations (20), (21) and (28) must be replaced by + , and that the drag correction factor $(\kappa(1 / 2 \tau, 0)$ of [24]) is

$$
\begin{aligned}
\Lambda(-\tau) & =1+\varepsilon_{1}+\varepsilon_{1} \varepsilon_{2}+\varepsilon_{1} \varepsilon_{2} \varepsilon_{3}+\cdots \\
& ={ }_{2} \Phi_{1}\left(y^{2}, y^{2} ; y^{4} ;+y\right) \\
& =\frac{1-y^{2}}{y}\left\{\frac{y}{1-y^{2}}+\frac{y^{2}}{1-y^{4}}+\frac{y^{3}}{1-y^{6}}+\cdots\right\} \\
& =\frac{1-y^{2}}{y}\left\{\left(\frac{y}{1-y}+\frac{y^{2}}{1-y^{2}}+\frac{y^{3}}{1-y^{3}}+\cdots\right)\right. \\
& \left.-\left(\frac{y^{2}}{1-y^{2}}+\frac{y^{4}}{1-y^{4}}+\frac{y^{6}}{1-y^{6}}+\cdots\right)\right\} \\
& \sim \sinh (\alpha)\left\{\frac{\gamma-\ln \frac{1}{2} \alpha}{\alpha}+\sum_{j=1}^{\infty} \frac{\left(2^{2 \jmath}-2\right)\left(B_{2 \jmath}\right)^{2} \alpha^{2 \jmath-1}}{2 j(2 j) !}\right\} \\
& =\sinh (\alpha)\left\{\frac{0.57721 . .-\ln \frac{1}{2} \alpha}{\alpha}+\frac{\alpha}{72}+\frac{7 \alpha^{3}}{43200}+\frac{31 \alpha^{5}}{3810240}+\cdots\right\}
\end{aligned}
$$

[7], where the asymptotic series is valid for $\tau \rightarrow \frac{1}{2}-$, i.e., $y \rightarrow 1-$ or $\alpha \rightarrow 0+$.

The bubble velocity, which is proportional to $1 / \Lambda(-\tau)$, remains close to $1-\tau$ until the bubble is very near the surface, being only $6 \%$ below that value when $\tau=0.4$, i.e., the bubble's topmost point is $\frac{1}{4}$ of its radius below the surface. Beyond that point the velocity decreases rapidly towards zero as $\tau$ increases. This theory would in practice be rather inaccurate so close to a free surface, because the bubble would push up the surface above $[1,3,15,17,18]$. The same objection holds for the theory of Wacholder and Weihs also. The distortion of the plane 
surface is impeded by gravity, and will be small if the parameters $\eta U / \sigma, \eta U / \rho g a^{2}$ are small, [3], where $\rho$ is the density of the fluid and $g$ the acceleration due to gravity.

\section{A line of equally spaced bubbles}

Suppose a line of $n$ bubbles each of radius $a$, initially equally spaced, is set free to rise in a liquid. The resultant force on each is the same, but the velocities $V_{1}$ will vary along the line, where $1 \leqslant i \leqslant n$. As in the previous theory, each bubble will have a primary Stokeslet of strength $4 \pi \eta a V$, at its centre provided that the motion is quasi-steady (changing significantly slower than vorticity diffuses). Every Stokeslet will have an image in every bubble except the one which it is inside; a computer is needed to carry the calculation of image strengths and positions to high order, because the number of images multiplies itself by $(n-1)$ at each stage. (For rigid instead of shear-free surfaces the number of images increases faster even than this, [10], which requires methods other than image analysis to be used $[11,17]$.) Finally, one solves for the $V$, by requiring the total sum of image Stokeslets inside each bubble to be the resultant force exerted by it on the fluid, i.e, the gravitational upthrust $F=(4 / 3) \pi \rho g a^{3}$. Convergence is tested by carrying the calculation up to varying orders of images; all $n(n-1)^{k-1}$ image Stokeslets of any given order $k$ are of the same sign, and opposite to that of the next order, and so the $V$, oscillate towards their final values as the order is increased. Gluckman et al. [11] instead assumed equal velocities (for rigid spheres in line) and calculated forces varying along the line.

TABLE 2. Speeds of the various bubbles in a line, the unit being the speed of an isolated bubble of the same radius in the same fluid. The unit of spacing is the bubble radius. The distribution of velocities about the middle of an equally spaced line is symmetrical; only the top half is given.

\begin{tabular}{ccccc}
$\begin{array}{c}\text { Number of } \\
\text { bubbles }\end{array}$ & $\begin{array}{c}\text { Position } \\
\text { in line }\end{array}$ & Spacing & Speed & $\begin{array}{c}\text { First-order } \\
\text { theory for speed }\end{array}$ \\
\hline 3 & 1 & 2.5 & 1.5861 & 1.6000 \\
4 & 2 & & 1.7296 & 1.8000 \\
& 1 & 4 & 1.4580 & 1.4583 \\
5 & 2 & & 1.6164 & 1.6250 \\
& 1 & 6 & 1.3474 & 1.3472 \\
8 & 2 & & 1.4708 & 1.4722 \\
& 3 & \multirow{2}{*}{10} & 1.4988 & 1.5000 \\
& 1 & & 1.2593 & 1.2593 \\
& 2 & & 1.3449 & 1.3450 \\
& 3 & & 1.3782 & 1.3783 \\
& 4 & &
\end{tabular}


Table 2 shows the results for several typical cases, and also the "first-order" approximation of taking the velocity of each bubble to be $F / 4 \pi \eta a$ increased by the Stokeslet velocity $F / 4 \pi \eta r_{i}$ of each other bubble at a distance $r_{1}$ away from it. That approximation turns out to be very good, in fact better than for the lines of rigid spheres for which it was first suggested [22], and better than the alternative which has been used [19], of considering only nearest-neighbour interactions. Table 2 gives also the closest bubble spacing for which our process converged reliably in a reasonable amount of computer time. In each case investigated that was a number of radii of the same order as the number of bubbles. For bubbles further apart than the spacing in the table the first-order approximation was even better.

Morrison's overall result [19] is unaffected by the roughness of his approximation. The second bubble in a line rises faster than the first, catches it up, and henceforth the pair moves upwards together and faster still (see Section 4 above). Then the third bubble finds itself at the top of the line, and the fourth moves up to it in the same way, and so on.

\section{Conclusions}

The image system of an axisymmetric Stokes flow in a shear-stress-free sphere with its centre on the axis of symmetry has been presented. If the original flow is from a Stokeslet due, as usual, to a force exerted at a point in the fluid, the image is just one additional Stokeslet. When there are two spherical boundaries, the higher-order images can be calculated with rather less trouble than the expansions in spherical harmonics previously used to solve such flow problems. A bubble rising vertically in a fluid beneath either another bubble or a horizontal surface is one case in point, for which the simpler series has permitted alternative analytical forms to be recognized, including a closed form and an asymptotic expansion.

A second sphere theorem shows that shear-free spherical stream surfaces invert with respect to any point on the axis into other surfaces of the same kind. This theorem was useful in computing the streamline pattern for flow past a pair of touching bubbles, where more direct methods converged too slowly to be useful. It suggests finding the properties of flow past unequal bubbles by first inverting with respect to a point which makes their inverses have the same radius. The theorem also holds for rigid surfaces, and simplifies the calculations for the images of point singularities in them.

One can also calculate the velocity of each of a line of rising bubbles. A good approximation is that each bubble rises at the speed it would in an infinite fluid, plus the speed induced by each other bubble treated as a single central Stokeslet. 


\section{Acknowledgements}

I am grateful to A. A. Lacey, T. R. F. Nonweiler and G. C. Wake who helped me find various properties of the function $\Lambda(\tau)$.

\section{References}

[1] K. Aderogba and J. R. Blake, "Action of a force near the planar surface between two semi-infinte immiscible liquids at very low Reynolds numbers", Bull. Austral. Math. Soc. 18 (1978), 345-356, and 19 (1979), 309-318.

[2] W N Bailey, Generaltsed hypergeometric series (Cambridge University Press, 1932).

[3] C. Berdan and L. G. Leal, "Motion of a sphere in the presence of a deformable interface, I, Perturbation of the interface from flat: the effects of drag and torque. II, A numerical study of the translation of a sphere normal to an interface", J. Collord Interface Sci. 87 (1982), 62-80 and 81-106.

[4] J. R Blake and A. T. Chwang, "Fundamental singularities of viscous flow. Part I: The image systems in the vicinity of a stationary no-slip boundary", J. Engrg. Math. 8 (1974), 23-29.

[5] J. C. Burns, "A generalization of Milne-Thomson's circle theorem", J. Math. Phys. Sci. 7 (1973), 373-382.

[6] S. F J. Butler, "A note on Stokes' stream function for motion with a spherical boundary", Proc. Cambridge Philos. Soc. 49 (1953), 169-174.

[7] T. J. I'A. Bromwich, An introduction to the theory of infinite series, 2nd ed. (Macmillan, 1926), p. 387.

[8] A. T. Chwang and T. Y. T. Wu, "Hydromechanics of low-Reynolds number flow. Part 2. Singularity method for Stokes flows", J. Fluid Mech. 67 (1975), 787-815.

[9] W. D. Collins, "A note on Stokes' stream-function for the slow steady motion of viscous fluid before plane and spherical boundaries", Mathematika l (1954), 125-130.

[10] W. D Collins, "Note on a sphere theorem for the axisymmetric Stokes flow of a viscous fluid", Mathematika 5 (1958), I18-121

[11] M. J. Gluckman, R. Pfeffer and S. Weinbaum, "A new technique for treating multiparticle slow viscous flow: axisymmetric flow past spheres and spheroids", J. Fluad Mech. 50 (1971), $705-740$.

[12] E. R. Hansen, A table of series and products (Prentice-Hall, Englewood Cliffs, 1975).

[13] J. Happel and H. Brenner, Low Reynolds number hydrodynamics (Prentice-Hall, Englewood Cliffs, 1965).

[14] J. F. Harper, "The motion of bubbles and drops through liquids", Adv. Appl. Mech. 12 (1972), $59-129$.

[15] S. Hartland, "The effect of circulation patterns on the drainage of the film between a liquid drop and a deformable liquid-liquid interface", Chem. Engrg. Sci. 24 (1969), 611-613.

[16] G. Hetsroni and S. Haber, "The flow in and around a droplet or bubble submerged in an unbounded velocity field", Rheol. Acta 9 (1970), 488-496.

[17] S. H. Lee, R. S. Chadwick and L. G. Leal, "Motion of a sphere in the presence of a plane interface. Part I. An approximate solution by generalization of the method of Lorentz". J. Fluid Mech. 93 (1979), 705-725.

[18] S. H. Lee and L. G. Leal, "Motion of a sphere in the presence of a plane interface. Part II. An exact solution in bipolar coordinates", J. Fluid Mech. 98 (1980), 193-224.

[19] F. A. Morrison, Jr., "Breakup of a bubble chain", Chem. Engrg. Sct. 28 (1973), 1115-1116. 
[20] S. D. Nigam and V. Srinivasan, "No-slip images in a sphere", J. Math. Phys. Sc. 9 (1975), 389-398.

[21] D. Pedoe, A course of geometry for colleges and universities (Cambridge University Press, 1970).

[22] H. R. Pruppacher and J. D. Klett, Microphysics and clouds and precipitation (Reidel, Dordrecht. 1978), p. 636.

[23] R. Usha, "Flow at small Reynolds numbers", Ph. D. Thesis, Indian Institute of Technology, Madras, 1980.

[24] E. Wacholder and D. Weihs, "Slow motion of a fluid sphere in the vicinity of another sphere or a plane boundary", Chem. Engrg. Sci. 27 (1972), 1817-1828. 